\title{
LA LEY DE LA RADIACIÓN NEGRA Y SU RELACIÓN CON LA FUNCIÓN ZETA DE RIEMANN
}

\section{THE LAW OF BLACK RADIATION AND ITS RELATIONSHIP WITH THE ZETA FUNCTION OF RIEMANN}

\author{
Roosevelt Carrillo Martínez ${ }^{1}$;;Huberto Barrios Escobar².;Julio Enrique \\ Duarte $^{3}$ \\ ${ }_{1}^{1}$ Departamento de Física. Universidad Popular del Cesar. rooseveltcarrillo@unicesar.edu.co \\ 2 Departamento de Matemáticas y Estadística. Universidad Popular del Cesar. \\ hbarrios@unicesar.edu.co \\ 3 Escuela Educación Industrial. Universidad Pedagógica y Tecnológica de Colombia. Sede \\ Duitama. julioenriqued1@gmail.com
}

\section{Resumen}

En la ley de radiación térmica, encontrada por Max Plank, para dar explicación teórica a la curva de radiación que habían encontrado los experimentalistas para la radiancia espectral y las leyes de radiación implícitas en dicha radiancia, se encuentra con una integral que conduce a la función Zeta de Riemann y la ley de Stefan-Boltzmann que se cumple en la radiación espectral del cuerpo negro. Se parte de la ley de distribución de Boltzmann; a continuación se presenta la formulación matemática de Planck de la radiación para luego llegar a la solución de dicha ecuación. En este desarrollo se muestra cómo se pueden entrelazar dos principios: uno físico, la ley de radiación negra, con uno matemático, la función zeta de Riemann, y llegar a la deducción de otro principio fundamental: la constante universal de radiación.

Palabras Clave: Radiancia Espectral, Ley de Stefan Boltzmann, Función Gamma, Función Zeta de Riemann.

\section{Abstract}

In the law of thermal radiation, found by Max Plank, to give a theoretical explanation to the radiation curve that the experimentalists had found for the 
spectral radiance and the radiation laws implicit in said radiance, one finds an integral that leads to the function Zeta de Riemann and the law of StefanBoltzmann that is fulfilled in the spectral radiation of the black body. It is based on Boltzmann's distribution law; Next, Planck's mathematical formulation of radiation is presented to then arrive at the solution of said equation. This development shows how two principles can be intertwined: a physical one, the black radiation law, with a mathematical one, the Riemann zeta function, and arrive at the deduction of another fundamental principle: the universal radiation constant.

Key Words: Spectral Radiance, Stefan Boltzmann's Law, Gamma Function, Zeta Riemann Function.

\section{INTRODUCCIÓN}

La Física es el campo de conocimiento científico de más rápido desarrollo que existe. No se tiene un conjunto limitado de leyes fundamentales que podamos resumir en una página y con ellas mostrar cómo operan en todas las circunstancias posibles. Aún no se conocen todas las leyes Fundamentales. Cada parte del todo del mundo real que hoy conocemos es solo una cierta forma de aproximación a la verdad completa, o la verdad completa hasta donde la conocemos (Duarte et al., 2013).

La enseñanza de las ciencias, y en especial de la Física, implica reconocer que el conocimiento científico no es absoluto ni dogmático, sino que se mantiene estable en la medida en que no aparezcan hechos o fenómenos que no puedan ser explicados por la teoría vigente (Zuluaga, 2017). En otras palabras, la búsqueda de una explicación más certera de la realidad es la razón para que los científicos estén siempre proponiendo nuevas teorías y modelos, ampliando con ello las fronteras del conocimiento. Un mejor entendimiento de los fenómenos físicos y naturales, a través de modelos que permitan explicar y predecir su comportamiento, impulsan la aparición de nuevas tecnologías y la evolución de las existentes, junto con cambios en el contexto social (Martínez et al., 2013; Reyes et al., 2016).

Un problema fundamental que tenían los físicos al final del siglo XIX era el de encontrar la ley de radiación térmica partiendo del principio microscópico de la materia, no fenomenológico ni empírico. Ellos construyeron un modelo ideal de radiación, Ilamada radiación de cuerpo negro. Consideraron una cavidad cuyas paredes están a cierta temperatura $T$ en equilibrio térmico con la radiación. Los 
átomos que componen las paredes emiten radiación electromagnética y absorben la radiación emitida por otros átomos de las paredes de la cavidad. El campo de la radiación ocupa todo el volumen de la cavidad radiante. Cuando la radiación encerrada alcanza el equilibrio térmico con las paredes, la densidad de energía del campo electromagnético es estacionaria, es decir, que no varía con el tiempo.

Según esta nueva concepción de la mecánica, la radiación, caracterizada anteriormente por su continuidad, se reducía a gránulos materiales (cuantos) o cantidades discretas de energía. No obstante, al definir estados estacionarios del electrón, se le atribuía a éste un simultáneo carácter ondulatorio: a la cantidad de movimiento del electrón había que hacer corresponder una longitud de onda, con lo cual la constante de Planck, que había servido para introducir el carácter corpuscular en la teoría de la radiación, permitía trasladar también la naturaleza ondulatoria a los corpúsculos materiales.

En equilibrio, la radiación electromagnética encerrada tiene una ley de distribución de energía bien definida. Lo que significa que a cada frecuencia (color) corresponde una densidad de energía que depende únicamente de la temperatura de las paredes, $T$, y es independiente de las características del material de la cavidad. La densidad de energía radiada para un rango de frecuencia entre $v$ y $v+d v$ es $E(v) d v$, donde $E(v)$ es la energía por intervalo unitario de frecuencias a una temperatura $T$. Encontrar la forma explícita de $E(v)$ era lo que ocupaba a los físicos de los finales del siglo $X I X$. El problema de encontrar los mecanismos por los cuales los átomos radiantes absorben y emiten radiación, es decir, encontrar la distribución de energía de la radiación de cuerpo negro dio lugar a la mecánica cuántica, (MC) hacia finales del siglo XIX. Todas las tentativas de encontrar una distribución de energía habían fracasado, debido a que usaban conceptos conocidos afianzados clásicamente como principios, uno de ellos era el de la aparente continuidad de la materia y la energía.

El presente documento presenta la relación que ha existido siempre entre las dos ciencias más fundamentales conocidas hasta hoy, la física y la matemática. Iniciando con una breve cronología de la mecánica cuántica, se establece la base para ir introduciendo los fundamentos matemáticos que dieron origen a esta teoría. Se parte de la ley de distribución de Boltzmann; a continuación se presenta la formulación matemática de Planck de la radiación para luego llegar a la solución de dicha ecuación. En este desarrollo se muestra cómo se pueden entrelazar dos principios: uno físico, la ley de radiación negra, con uno matemático, la función 
zeta de Riemann, y llegar a la deducción de otro principio fundamental: la constante universal de radiación.

\section{CRONOLOGÍA DE LA MECÁNICA CUÁNTICA}

Resulta paradójico el hecho de que el electrón y el neutrón fueran descubiertos en 1987 y 1932 respectivamente y que la que probablemente es una de las disciplinas ligadas a ellos, la MC, comenzase su desarrollo más temprano, por allá en 1859. Gustav Kirchoff en ese año publico unas teorías sobre la radiación del cuerpo negro en las cuales relacionaba la energía irradiada con la temperatura y la frecuencia de la energía emitida. Esto quedaba probado, pero él fue incapaz de encontrar esa relación, y la función de esa energía irradiada toma la forma de $E=$ $J(T, v)$ siendo $J$ una función respecto a la temperatura y el tiempo que era desconocida. A partir de entonces fueron múltiples los intentos en descubrir la forma de dicha función $J$.

Uno de los primeros intentos con algo de éxito fue protagonizado por Josef Stefan, el cual, de forma experimental, concluye que la energía emitida se relacionaba con la cuarta potencia de la temperatura. A esta misma conclusión llego de forma teórica Ludwig Boltzmann, aplicando la termodinámica y los principios electromagnéticos de Maxwell. Estas conclusiones eran buenas, pero no respondían al problema de longitudes de onda específicas, así pues, no estaban completas.

En 1896, Wilhelm Wien propuso una teoría que encajaba perfectamente para valores bajos de la longitud de onda, pero fallaba más allá del infrarrojo como probaron Rubens y Kurlbaum. No fue hasta 4 años más tarde cuando Planck fue visitado por Rubens que le enseño sus estudios. Poco tiempo después Planck había desarrollado la parte matemática del problema y había descrito con gran exactitud lo que debía ser la función J. Pero esto no contento a Planck, de manera teórica sus resultados y dicha función $J$ solo se explicaban de una forma, la energía era emitida en cantidades indistinguibles llamadas cuantos.

En 1888, tras la muerte de Kirchoff, Planck ocupo su lugar en la universidad de Berlín avalada por su adquirido prestigio como físico y sus visiones originales de la ciencia en general. Mientras tanto continuó su estudio de la termodinámica y la emisión de energía en función de la longitud de onda de la misma. Esto le llevaría en 1900 a publicar una formula hoy en día conocida como la fórmula de radiación de Planck. En dos meses Planck hizo un completo estudio teórico-ético en el cual introducía un concepto rompedor con la física conocida hasta aquel instante, el 
cuanto de energía. A finales de dicho año Planck expuso sus teorías públicamente con el tema del cuanto como mayor fuente de controversia y la aceptación del carácter estadístico de las leyes físicas interpretado por Boltzmann, quien estableció la conocida ley de distribución para los gases:

$p(\epsilon)=\frac{1}{K T} e^{-\frac{E}{K T}}$

Donde

$\epsilon$ = energía de una partícula

$T=$ temperatura absoluta

$K=$ constante de Boltzmann

La radiación se asumió como si fuera un gas ideal con energía continua y se aplicó la distribución de Boltzmann para encontrar la energía media por cada ente, o partícula, radioactivo de frecuencia $v$, lo cual implica realizar una integral sobre todo el intervalo de energía que va desde cero al infinito, como se muestra a continuación:

$\bar{\epsilon}=\frac{1}{K T} \int_{0}^{\infty} \epsilon e^{-\frac{E}{K T}} d \epsilon$

El valor de esta integral es $K T$, la cual es conocida como Teorema de Equipartición de la Energía. Se encontró que todas las ondas electromagnéticas estacionarias tenían el mismo valor $K T$ independiente de la longitud de onda de la radiación atrapada por la cavidad. Este resultado llevo a una conclusión absurda: una radiación monocromática de energía arbitraria, por absorción y emisión sucesiva, va convirtiéndose rápidamente en una cantidad infinita de longitudes de onda cada vez más cortas. Esto conlleva abiertamente a una violación del principio fundamental de conservación de la energía, puesto que da como resultado que la cavidad tendría energía infinita.

\section{FORMULACIÓN DE PLANCK DE LA RADIACIÓN}

En 1900, el físico alemán Max Karl Ernst Ludwig Planck encontró la formula correcta de radiación. Para ello tuvo que romper con el pensamiento común de la continuidad de la energía, idea que él mismo se resistía a admitir; pero fue Albert Einstein quien, no solo la admitiría, sino que la elevaría a nivel de principio físico 
fundamental, el de la cuantización de la energía, en paquetes energéticos para los cuales surgió la palabra "fotón".

Planck sugirió que la radiación dentro de la cavidad está en equilibrio con los átomos, y debe haber una relación funcional entre la distribución de energía y la energía absorbida o emitida de los átomos. Planck supuso como modelo para los átomos que ellos se comportan como osciladores armónicos de frecuencia $v$ y como hipótesis fundamental que cada oscilador puede absorber o emitir energía radiante en paquetes discretos proporcionales a la frecuencia $v$ : $\epsilon_{n}=n h v ; n=1,2,3 \ldots$; donde $h$ es la constante de Planck igual a $6,62 \times 10^{-34} \mathrm{Js}$. Este supuesto rompe con el principio clásico de la continuidad de la energía.

La cuantización de la energía, y de otras magnitudes, se considera como principio fundamental. Con esta nueva idea, y con la ley de reparto de Boltzmann (Martínez, 1999) ahora, tomándose en forma discreta, Planck entra a calcular la nueva forma de radiación. Lo que sintetizó en la siguiente ecuación, la cual es la nueva función de distribución de la energía:

$p\left(\epsilon_{i}\right)=\frac{1}{Z} e^{-\frac{\epsilon_{i}}{K T}}$

donde:

$Z=\sum_{i=1}^{\infty} e^{-\frac{\epsilon_{i}}{K T}}$

$p\left(\epsilon_{i}\right)=$ probabilidad de que el sistema tenga energía $\epsilon_{i}$, siendo la energía del ente, o partícula, $i$.

La energía media es, utilizando la cuantización de Planck, $\epsilon_{n}=n h v$

$\bar{\epsilon}=\frac{\sum_{i=1}^{\infty} \epsilon_{i} e^{-\frac{\epsilon_{i}}{K T}}}{Z}=\frac{\sum n h e^{-\frac{n h v}{K T}}}{\sum e^{-\frac{n h v}{K T}}}$

Si se hace

$\alpha=\frac{h v}{K T}$

Entonces la energía media se puede expresar: 
$\bar{\epsilon}=\frac{\sum n h e^{-\frac{n \alpha}{K T}}}{\sum e^{-\frac{n \alpha}{K T}}}$

Nuevamente, si se realiza la siguiente sustitución $r=e^{-\alpha}$, en la ecuación de la energía media, se obtiene:

$\bar{\epsilon}=\alpha K T \frac{\sum_{n=0}^{\infty} n r^{n}}{\sum_{n=0}^{\infty} r^{n}}$

Puesto que $|r|<1$, las series geométricas $\sum_{n=0}^{\infty} r^{n}$ y $\sum_{n=0}^{\infty} n r^{n}$ tienen como sumas,

$s=\sum_{n=0}^{\infty} r^{n}=\frac{1}{1-r}$

$\sum_{n=0}^{\infty} n r^{n}=r \frac{d}{d r} \sum_{n=1}^{\infty} r^{n-1}=\frac{r s}{1-r}$

Sustituyendo (3) en (1) se establece que

$\bar{\epsilon}=h v \frac{e^{\frac{h v}{K T}}}{1-e^{\frac{h v}{K T}}}=h v \frac{1}{e^{\frac{h v}{K T}}-1}$

De la ecuación (4) se observa que la energía media depende no solo de la temperatura $T$, sino también de la frecuencia $v$ radiada y tiende rápidamente a cero cuando $v$ tiende a más infinito.

\section{SOLUCIÓN DE LA ECUACIÓN DE RADIACIÓN}

Ya se sabía, de la física estadística, que el valor medio $\bar{\epsilon}(v)$ hay que multiplicarlo por todas las maneras posibles (entes) presentes de frecuencia $v$ y $v+d v$, lo que nos conduce a la distribución de radiación siguiente:

$\rho(v, T)=g(v) \bar{\epsilon}(v)=\left[\frac{8 \pi}{c^{3}} v^{2}\right] \bar{\epsilon}(v)$

De las ecuaciones (3) y (4), haciendo manipulaciones algebraicas, se llega a:

$\rho(v, T)=\left[\frac{8 \pi h}{c^{3}}\right] \frac{v^{3}}{e^{\frac{h v}{K T}}-1}$ 
A la ecuación (5) se le conoce como la ley de radiación de cuerpo negro. La cual explica las leyes empíricas antes conocidas: la de Stefan-Boltzmann, la de Wien y la curva experimental de radiación obtenidas antes de Planck.

Integrando (5) desde cero hasta más infinito se obtiene la energía total radiada por unidad de volumen, la cual se sabía empíricamente que era proporcional a la cuarta potencia de la temperatura, es decir, $E_{\text {Total }}=\sigma T^{4}$. La constante tiene el valor de:

$\sigma=5,67 \times 10^{-8} \mathrm{~W} / \mathrm{m}^{2} K^{4}$

Este resultado se manifiesta automáticamente integrando a (5), esto es:

$E(v)=\int_{0}^{\infty} \rho(v, T) d v=\left[\frac{8 \pi h}{c^{3}}\right] \int_{0}^{\infty} \frac{v^{a}}{e^{\frac{h v}{K T}-1}} d v$

Si se realiza el siguiente cambio de variable $x=\frac{h v}{K T}$ en (6), se llega al siguiente resultado:

$E(v)=\left[\frac{8 \pi h}{c^{3}}\right] \frac{K T}{h} \int_{0}^{\infty} \frac{x^{3}}{e^{x}-1} d v$

Para la solución a (7) considérese la integral

$I(k)=\int_{0}^{\infty} \frac{x^{k-1}}{e^{x}-1} d v$

donde $k$ es un entero positivo.

Multiplicando al numerador y al denominador del integrando, en la ecuación (8), por $e^{-x}$ se obtiene:

$I(k)=\int_{0}^{\infty} \frac{x^{k-1} e^{-x}}{1-e^{-x}} d x$

Puesto que $0<e^{-x}<1$ para $x \in(0,+\infty)$. La serie geométrica

$1+\left(e^{-x}\right)+\left(e^{-x}\right)^{2}+\left(e^{-x}\right)^{3}+\left(e^{-x}\right)^{4}+\cdots=\frac{1}{1-e^{-x}}$

Es decir, se tiene 
$1+\left(e^{-x}\right)+\left(e^{-2 x}\right)+\left(e^{-3 x}\right)+\left(e^{-4 x}\right)+\cdots=\frac{1}{1-e^{-x}}$

Si se reemplaza (3) en (2) se obtiene

$\int_{0}^{\infty} \frac{x^{k-1} e^{-x}}{1-e^{-x}} d x=\int_{0}^{\infty} x^{k-1} e^{-x}\left(1+e^{-x}+e^{-2 x}+e^{-3 x}+e^{-4 x}+\cdots\right) d x$

Por lo tanto

$I(k)=\int_{0}^{\infty} x^{k-1} e^{-x} d x+\int_{0}^{\infty} x^{k-1} e^{-2 x} d x+\int_{0}^{\infty} x^{k-1} e^{-3 x} d x+\cdots$

Si se realiza la sustitución $u=n x$ se llega a la siguiente igualdad:

$\int_{0}^{\infty} x^{k-1} e^{-n x} d x=\frac{1}{n^{k}} \int_{0}^{\infty} x^{k-1} e^{-u} d u$

Pero la integral del segundo miembro de (5) es una función Gamma (Apostol, 1977). Es decir,

$\Gamma(k)=\int_{0}^{\infty} x^{k-1} e^{-u} d x=(k-1) !$

donde $k$ es un número entero no negativo.

En particular, si k es un número entero no negativo se concluye de (5) que

$\int_{0}^{\infty} x^{k-1} e^{-n x} d x=\frac{(k-1) !}{n^{k}}$

Así, si se sustituye (6) en cada uno de los sumandos de (4) se adquiere la siguiente igualdad:

$I(k)=(k-1) !+\frac{(k-1) !}{2^{k}}+\frac{(k-1) !}{3^{k}}+\frac{(k-1) !}{4^{k}}+\cdots=(k-1) ! \sum_{n=1}^{\infty} \frac{1}{n^{k}}$

En particular, para $k=4$ se tiene que la suma de la función Zeta de Riemann (Apostol, 1977) (Calderón, 2002) es:

$\zeta(4)=\sum_{n=1}^{\infty} \frac{1}{n^{4}}=\frac{\pi^{4}}{90}$

Si se reemplaza (15) en (7) se llega a 
$E(T)=\left[\frac{8 \pi^{5} K^{4}}{15 h^{3} c^{3}}\right] T^{4}$

Donde la cantidad en corchete es el valor teórico de la constante universal, que habían encontrado de manera empírica Stefan y Boltzmann independientemente, para la teoría de radiación y la termodinámica de gases (Kuhn, 1983) (Einstein, 1965).

Usando dos enfoques, primero uno físico y luego uno matemático, se ha llegado a un resultado de vital importancia en la comprensión de un fenómeno físico como lo es la radiación del cuerpo negro. Es decir, partiendo de la fundamentación matemática de un concepto físico que luego se relaciona con una herramienta matemática, la función zeta de Riemann, se llega al valor de una de las constantes fundamentales de la ciencia física: la constante universal de radiación.

El estudio de la radiación dio origen a una de las teorías más exitosas hasta hoy: la mecánica cuántica. Además, la radiación es una energía que se propaga como una onda electromagnética, abarca todo el espectro que va desde las ondas de radio, pasando por las microondas, los rayos infrarrojos, la luz visible, los rayos ultravioleta, los rayos $\mathrm{x}$, rayos gamma y los rayos cósmicos. Otro campo de aplicación es el estudio de la ciencia de los materiales (Estrada-Rodríguez et al, 2017). Es muy bien sabido del basto campo de aplicación de todos estos fenómenos, e innegable los resultados experimentales que beneficiaron el nivel de vida de la población desde su descubrimiento hasta hoy. Decir que el tema está acabado es un atrevimiento y el campo investigativo, tanto teórico como experimental, sigue abierto.

\section{Conclusiones}

La percepción epistemológica del mundo se basa en explicaciones transitorias, al igual que otras teorías, como el círculo perfecto de las órbitas planetarias. Los conceptos de la materia y la energía mecánica y continua solo duraron alrededor de trescientos años, hubo que romperse ese paradigma junto con la matemática utilizada para el tratamiento de la continuidad. Así que, para encontrar las leyes de radiación, los conceptos clásicos quedaron obsoletos y debió inventarse una nueva manera de ver el mundo. Por lo tanto, fue necesario pasar de lo continuo a lo discreto.

El trabajo aquí presentado es una muestra clara que, con conceptos teóricos bien definidos en cualquier rama de las ciencias, se pueden entrelazar los puntos de 
convergencia entre los principios fundamentales comunes a ellas para presentarlos en diferentes escenarios donde se forman los científicos de hoy y del mañana; es decir, como ejercicio pedagógico es de vital importancia presentar varios enfoques de un mismo principio o ley.

\section{Referencias bibliogràficas}

(1).- Duarte, J., Reyes-Caballero, F., \& Fernández-Morales, F. (2013). La enseñanza de la física en los currículos de ingeniería. Revista de Investigación, Desarrollo e Innovación, 4(1), 45-55. Doi: http://dx.doi.org/10.19053/20278306.2606

(2).- Zuluaga-Duque, J. F. (2017). Relación entre conocimientos, saberes y valores: un afán por legitimar los saberes más allá de las ciencias. Revista de Investigación, Desarrollo e Innovación, 8(1), 61-76. doi: 10.19053/20278306.v8.n1.2017.5973

(3).- Martínez-Ovalle, S., Reyes-Caballero, F., \& González-Puin, L. X. (2013). Protección radiológica a trabajadores y público en instalaciones que operan radioisótopos industriales. Revista de Investigación, Desarrollo e Innovación, 3 (2), 120-124. doi: 10.19053/20278306.2166

(4).- Reyes-Caballero, F., Fernández-Morales, F., \& Duarte, J. (2016). Panorama energético. Revista de Investigación, Desarrollo e Innovación, 7(1), 151-163. Doi: http://dx.doi.org/10.19053/20278306.v7.n1.2016.5605

(5).- Martínez, R. (1999). La teoría de radiación de cuerpo negro. Momento, 19, 59-75.

(6).- Rudin, W. (1976). Principles of Mathematical Analysis. Third Edition, McGRAW-HILL.

(7) .-Apostol, T. M. (1977). Análisis Matemático. Segunda edición, Editorial Reverté.

(8).- Calderón, C. (2002). La función Z de Riemann. Revista Real Academia de Ciencias de Zaragoza, 57, 67-87.

(9).- Kuhn, T. S. (1983). La teoría del cuerpo negro y la discontinuidad cuántica, 1894-1912. Primera edición, Alianza Editorial.

(10).-Einstein, A. (1965). Concerning an Heuristic Point of View Toward the Emission and Transformations of Light. American Journal of Physics, 33(5). 
80

(11).- Estrada-Rodríguez, C. A., Parra-Vargas, C. A., Pimentel-Junior, J. L. (2017). Propiedades estructurales, eléctricas y magnéticas en el sistema $\mathrm{Na} 0.25$ Ba0.75CoO3. BISTUA Revista de la Facultad de Ciencias Básicas, 15 (1). doi: https://doi.org/10.24054/01204211.v1.n1.2017.2557

*Para citar este artículo: Carrillo Martínez R.;Barrios Escobar H; Duarte J E. The law of black radiation and its relationship with the zeta function of Riemann.Revista Bistua.2017.15(2):6980

+ Autor para el envió de correspondencia y la solicitud de las separatas: Carrillo Martínez R. Departamento de Física. Universidad Popular del Cesar. rooseveltcarrillo@unicesar.edu.co

Recibido: Noviembre 04 de 2016

Aceptado: Febrero 06 de 2017 